Published in final edited form as:

Angew Chem Int Ed Engl. 2008 ; 47(22): 4119-4121. doi:10.1002/anie.200800069.

\title{
Sensitive NMR Sensors Detect Antibodies To Influenza
}

\author{
Isaac Koh, Rui Hong, Ralph Weissleder, and Lee Josephson * \\ Center for Molecular Imaging Research Massachusetts General Hospital and Harvard Medical \\ School Building 149, 13th Street, Boston, MA 02129 (USA)
}

\section{Keywords}

antibodies; magnetic properties; peptides; biosensors; virus

Point of care (POC) sensors for the determination of immune status are an important part of the preparation for possible pandemics such as that created by avian influenza.[1,2] NMR based magnetic relaxation switches (MRSw's) are attractive for this application because they are indifferent to light and involve no immobilization of materials on vessel walls.[3] MR relaxometers are practical as POC sensors since their requirements for magnetic field strength, volume and homogeneity are minimal.[4-6]

To date, MRSw's have used magnetic nanoparticles (NPs) that react with molecular targets to aggregate and decrease $\mathrm{T}_{2}$. Although MRSw's detect highly multivalent viruses or bacteria with high sensitivity, $[7,8]$ their sensitivity for proteins is far lower. $[9,10]$ Our goal was to improve the sensitivity of MRSw sensors for divalent antibodies, so the immune status of birds or humans might eventually be determined using a POC MR relaxometer. A monoclonal antibody recognizing the Tag peptide from a hemagglutinin of a human influenza virus was used as a target to assess strategies for improving MRSw sensitivity.

An obvious strategy for improving MRSw sensor sensitivity was to employ the equivalence principle of antibody-antigen reactions involved in precipitin formation,[11-13] reducing the concentration of Tag peptide-magnetic particles (a synthetic multivalent antigen), so that lower concentrations of anti-Tag antibodies might achieve aggregation. The Tag peptide from the influenza hemagglutinin was therefore conjugated to nanoparticles (NPs) and far larger micronsized magnetic particles (MPs), to yield Tag-NPs and Tag-MPs. As indicated in Table 1, a TagMP contained 350,000 times more iron than a Tag-NP and had a correspondingly higher magnetic moment per particle. With a typical initial $\mathrm{T}_{2}$ for MRSw assays $(100 \mathrm{msec})$, the concentration of particles decreased from $2.8 \times 10^{-9} \mathrm{M}$ with Tag-NPs to $5.1 \times 10^{-15} \mathrm{M}$ using Tag-MPs.

Attempts to use Tag-MPs for MRSw assays indicated Tag-MPs differed from earlier NPs in two key respects. First, as shown in Figure 1, Tag-MP's underwent a reversible increase in $\mathrm{T}_{2}$ in the $0.47 \mathrm{~T}$ relaxometer magnet. Micrographs indicated the $\mathrm{T}_{2}$ increase was associated with MP aggregation resulting from magnetic attractions between Tag-MP's(see Figure 2 of

*corresponding author Fax: (+1)617-726-5708 E-mail: josephso@ helix.mgh.harvard.edu.

Justification

New sensors for the detection of antibodies to the structural features of viral proteins are an important part of the preparation for possible viral pandemics. Here we show that micron-sized particles that are 350,000 times more magnetic (per particle) than earlier magnetic nanoparticles provide a high sensitivity, NMR based sensor for detecting antibodies to influenza virus. A homogeneous magnetic field was employed to enhance antibody-based crosslinking between particles, providing a novel method of increasing the sensitivity of a homogeneous (non-separation based) immunoassay. 
reference [14]), with the randomizing effects of thermal energy causing dispersion when the field was removed. Second, Tag-MP aggregation resulted in a $\mathrm{T}_{2}$ increase while Tag-NP aggregation resulted in a $T_{2}$ decrease, $[3,15]$ observations consistent with the outer sphere diffusion theory used to describe the effects of magnetic particles on $T_{2}$.[16-19] This theory employs two parameters, $\tau_{d}$, the diffusion time for water, and $\Delta \omega$, the difference in angular frequency between the magnetic field experienced by a proton at the particle (or aggregate) surface versus that in the bulk. Outer sphere diffusion theory predicts $T_{2}$ will decrease as TagNPs aggregate, since the motional averaging condition is fulfilled with both dispersed and aggregated materials $\left(\Delta \omega * \tau_{d}<1\right)$. With Tag-MP aggregation, the resulting magnetic field inhomogeneities become so few and infrequent water molecules must traverse long distances to encounter them. Here the effects on $\mathrm{T}_{2}$ become diffusion limited $\left(\Delta \omega * \tau_{d}>1\right)$. The effects of NP and MP aggregation on $\mathrm{T}_{2}$ have been discussed.[14]

Three techniques were then explored separately and in combination to increase the sensitivity of MRSw assays, see Figure 2. First, a decrease in particle concentration was achieved by replacing the magnetic nanoparticle (NP) with a micron-sized particle (MP), see Figure 2a. The second technique, magnetic field enhanced, molecular target aggregation, exploited the reversible magnetic field induced MP aggregation in the absence of a target ligand shown in Figure 1. We hypothesized that the magnetic field aggregation of Tag-MPs might enhance antiTag mediated aggregation (Figure $2 \mathrm{~b}$ ). Therefore solutions of Tag-MPs were exposed to antiTag in the relaxometer magnet, resulting in both magnetic field induced and anti-Tag antibody mediated Tag-MP aggregation. Samples were then removed from the magnetic field, to allow Tag-MPs to disaggregate which occurred only in the absence of particle crosslinking by antiTag. $T_{2}$ was determined in less than 30 seconds by placing samples in the relaxometer so that magnetic field induced aggregation during $\mathrm{T}_{2}$ measurement was minimal. Finally, we employed valency enhancement, where the valency of the monoclonal anti-Tag was increased above the normal two antigen-combining sites per IgG by adding a sheep $\mathrm{F}\left(\mathrm{ab}^{\prime}\right)_{2}$ antibody to the $\mathrm{Fc}$ fragment of the mouse anti-Tag monoclonal antibody (Figure 2c).

We first measured $\mathrm{T}_{2}$ as a function of anti-Tag concentration using Tag-NPs and Tag-MPs (decreasing particle concentration but without magnetic field or valency enhancement) as shown in Figure 3a. Data were fit to a 4-parameter equation, to obtain the EC50 (midpoint), the Hill coefficient (curve slope), and computer generated maximum and minimum values of $\mathrm{T}_{2}$. Table 2 provides EC50's, Hill coefficients, and $\Delta \mathrm{T}_{2}$ 's for the curves shown in Figure 3a. $\Delta T_{2}$, the $T_{2}$ for the fully dispersed state minus the $T_{2}$ for the fully aggregated state, is positive for anti-Tag induced Tag-NP aggregation but negative for anti-Tag induced Tag-MP aggregation. $\% \Delta \mathrm{T}_{2}$ is the difference in $\mathrm{T}_{2}$ in the presence and absence of anti-Tag divided by $\Delta \mathrm{T}_{2}$ and expressed in percent.

Using Tag-NPs, increasing concentrations of anti-Tag gave an EC50 of $49 \mathrm{nM}$, versus an EC50 of $0.60 \mathrm{nM}$ for Tag-MPs. Further reductions in the EC50's of the MP based MRSw assays were obtained by the use of magnetic field and valency enhancement as shown in Figure 3b. Through a combination of all three techniques, the EC50's for anti-Tag were progressively decreased from a starting value of $49 \mathrm{nM}$ with Tag-NPs to $0.0020 \mathrm{nM}(2.0 \mathrm{pM})$, obtained with decreased particle concentration, magnetic field aggregation, and valency enhancement (Figure 3c).

To obtain a detection limit from these results, we determined a "projected sensitivity concentration" or PSC. Five msec was added to (or subtracted from) the value of $\mathrm{T}_{2}$ in the absence of anti-Tag and the concentration at this $\mathrm{T}_{2}$ determined from curve parameters (Table 2). The PSC for anti-Tag with Tag-NP sensor, the starting point of our investigation, was 26 $\mathrm{nM}$. By using our three techniques for enhancing sensitivity, the PSC was reduced to 0.00014 $\mathrm{nM}$ or 186,000 fold. The PSC is discussed further in Supporting Information. This detection 
sensitivity is comparable with those of most advanced nanostructure-based antibody assays. [20]

We obtained a high sensitivity MRSw sensor for influenza antibody by using MPs and exploiting a previously unrecognized feature of their response to a magnetic field. Application of a homogeneous magnetic field enhanced antibody-based crosslinking between particles, providing a novel method of increasing the sensitivity of a homogeneous, particle aggregation based immunoassay. Together with high throughput methods of peptide and peptide-MP conjugate synthesis,[21] MRSw sensors might be used, both to analyze the immune response to mutating viruses in laboratory settings, and for an MRSw based POC antibody sensor.

\section{Acknowledgements}

This work was supported by R01EB0004626 and U54CA119349.

\section{References}

1. Lu PS. Science 2006;312:337. [PubMed: 16627701]

2. Butler D. Nature 2006;439:248. [PubMed: 16421529]

3. Perez JM, Josephson L, O'Loughlin T, Hogemann D, Weissleder R. Nat. Biotechnol 2002;20:816. [PubMed: 12134166]

4. Manz B, Coy A, Dykstra R, Eccles CD, Hunter MW, Parkinson BJ, Callaghan PT. J. Magn. Reson 2006;183:25. [PubMed: 16891134]

5. Blumich B, Blumler P, Eidmann G, Guthausen A, Haken R, Schmitz U, Saito K, Zimmer G. Magn. Reson. Imaging 1998;16:479. [PubMed: 9803893]

6. Sillerud LO, McDowell AF, Adolphi NL, Serda RE, Adams DP, Vasile MJ, Alam TM. J. Magn. Reson 2006;181:181. [PubMed: 16698297]

7. Perez JM, Simeone FJ, Saeki Y, Josephson L, Weissleder R. J. Am. Chem. Soc 2003;125:10192. [PubMed: 12926940]

8. Kaittanis C, Naser SA, Perez JM. Nano Lett 2007;7:380. [PubMed: 17298004]

9. Kim GY, Josephson L, Langer R, Cima MJ. Bioconjugate Chem 2007;18:2024.

10. Perez JM, O'Loughin T, Simeone FJ, Weissleder R, Josephson L. J. Am. Chem. Soc 2002;124:2856. [PubMed: 11902860]

11. DeLisi C. J. Theor. Biol 1974;45:555. [PubMed: 4135952]

12. Chak KC, Hart H. Bull. Math. Biol 1980;42:37. [PubMed: 7357120]

13. van Oss CJ. J. Immunoassay 2000;21:143. [PubMed: 10929885]

14. Hong R, Cima MJ, Weissleder R, Josephson L. Mag. Reson. Med. 2008in press

15. Josephson L, Perez JM, Weissleder R. Angew. Chem. Int. Ed 2001;40:3204.

16. Gillis P, Koenig SH. Magn. Reson. Med 1987;5:323. [PubMed: 2824967]

17. Brooks RA. Magn. Reson. Med 2002;47:388. [PubMed: 11810684]

18. Yung KT. Magn. Reson. Imaging 2003;21:451. [PubMed: 12878254]

19. Roch A, Gossuin Y, Muller RN, Gillis P. J. Magn. Magn. Mater 2005;293:532.

20. Rosi NL, Mirkin CA. Chem. Rev 2005;105:1547. [PubMed: 15826019]

21. Weissleder R, Kelly K, Sun EY, Shtatland T, Josephson L. Nat. Biotechnol 2005;23:1418. [PubMed: 16244656]

22. Reynolds F, O'Loughlin T, Weissleder R, Josephson L. Anal. Chem 2005;77:814. [PubMed: 15679348] 


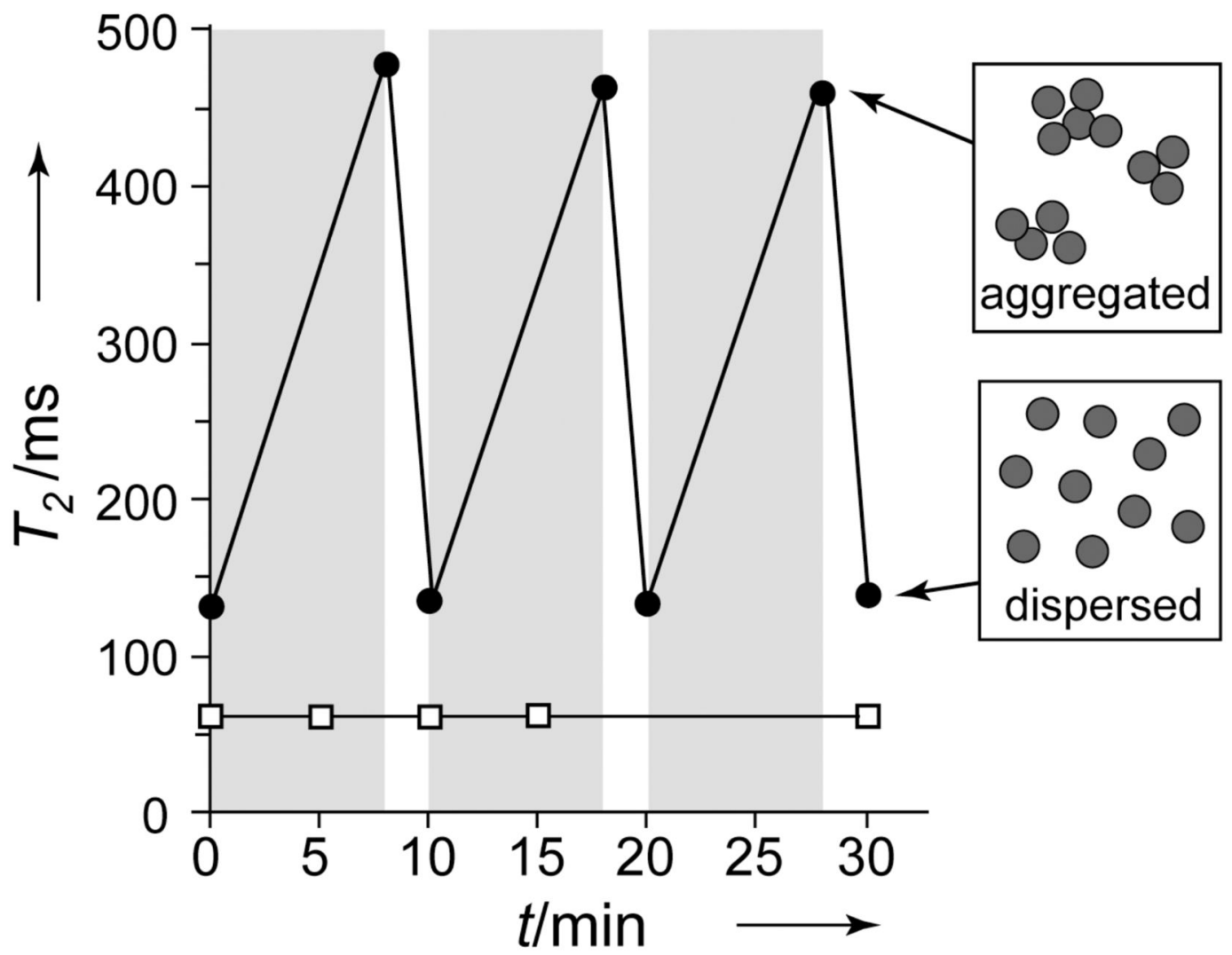

Figure 1. Magnetic particles respond to a homogeneous magnetic field

$\mathrm{T}_{2}$ of a MP solution ( $\bullet$ ), but not a NP solution ( $\square$ ), increases when in a $0.47 \mathrm{~T}$ field (gray). White, no field. 
a) Decreased Particle Concentration

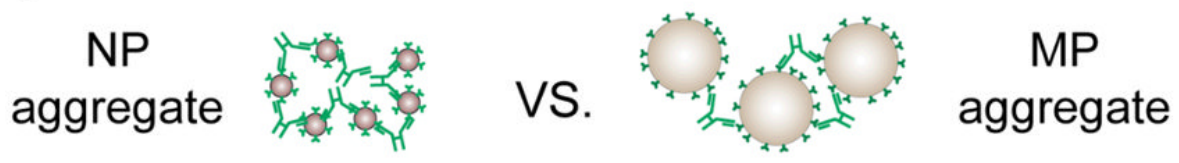

b) Magnetic Field Enhanced Target Aggregation

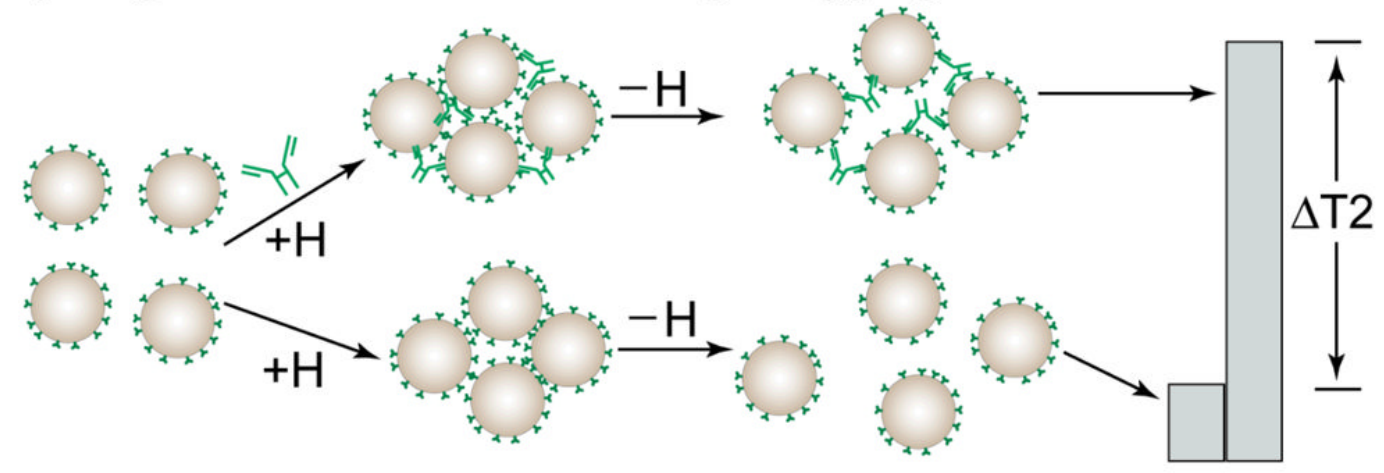

c) Valency Enhanced Aggregation

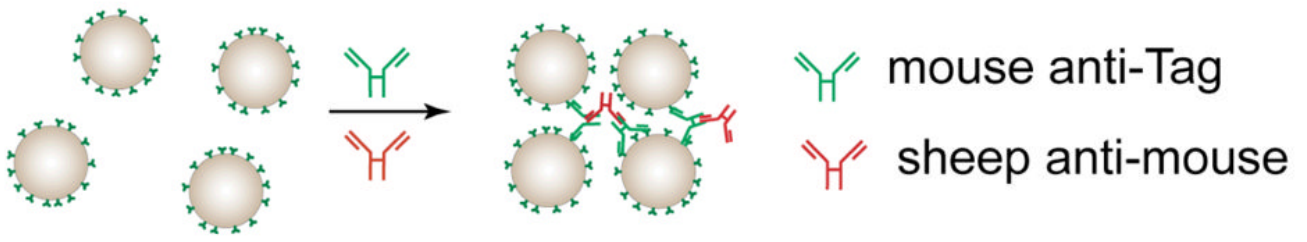

Figure 2. Methods of increasing MRSw sensitivity

a) Decrease in particle concentration. Anti-Tag forms bridges between larger MPs. b) Magnetic field enhanced target aggregation. A magnetic field $(+\mathrm{H})$ produces magnetic attractions between MPs that result in aggregation. In the presence of anti-Tag (top) the aggregate is maintained when the field is removed $(-\mathrm{H})$ and a high $\mathrm{T}_{2}$ results. c) Valency enhanced aggregation. The addition of an anti-Fc antibody permits anti-Tag to bind more than two MPs simultaneously. 

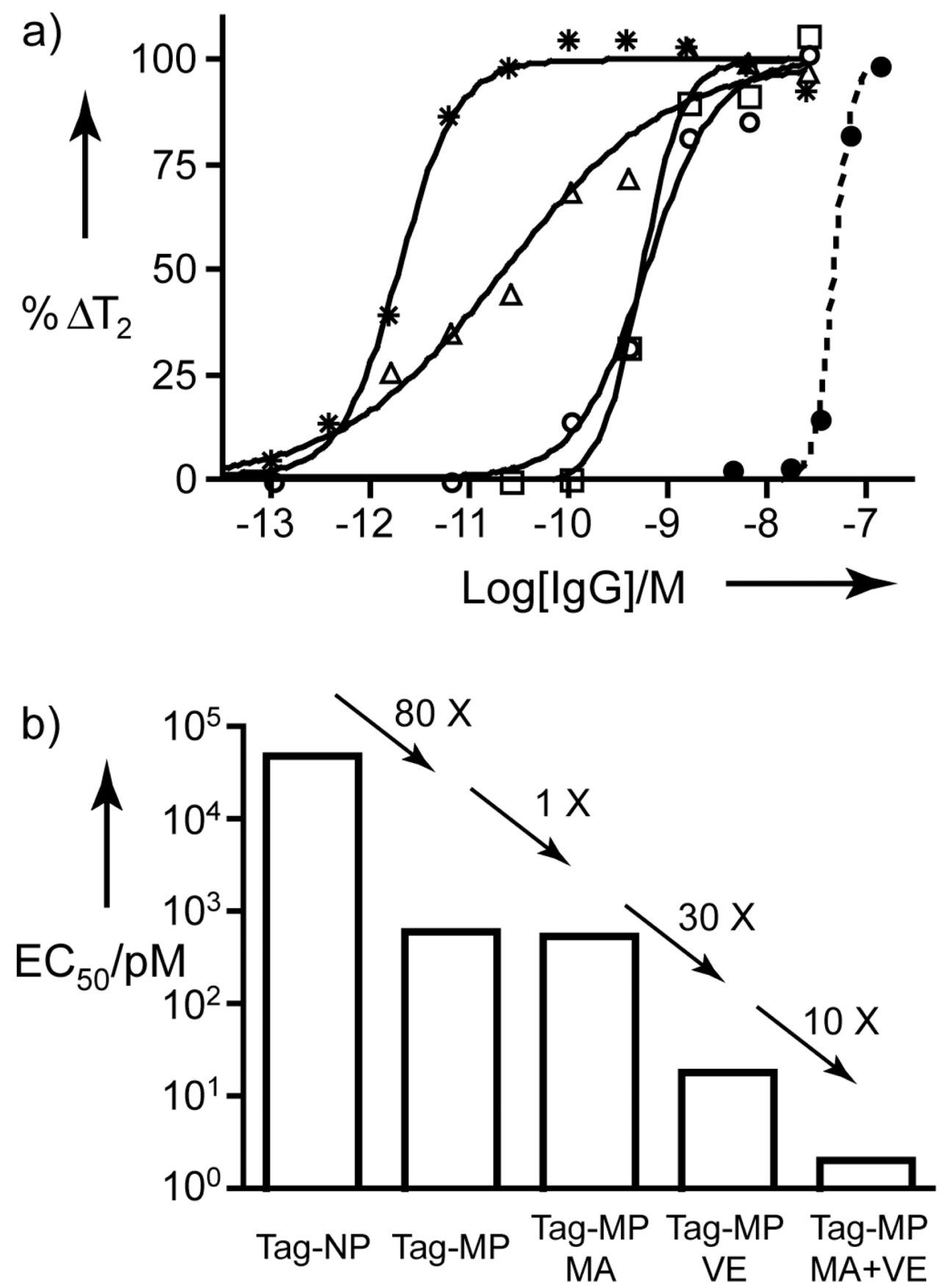

Figure 3. Techniques for increasing the response to anti-Tag antibody

a) The initial reference assay with Tag-NPs is shown with a dotted line and $(\bullet)$. To obtain a decrease in particle concentration, Tag-MPs $(\circ)$ were used. The Tag-MP was then used with magnetic field aggregation $(\square)$, with valency enhancement $(\Delta)$, and with both magnetic aggregation and valency enhancement ( $\lesssim$ ). b) EC50's obtained by decreasing particle concentration, magnetic field aggregation (MA), and valency enhancement (VE). Additional curve parameters are provided in Table 2. 
Table 1

Properties of magnetic particles (MPs) and nanoparticles (NPs).

\begin{tabular}{lll}
\hline Particle & MP & NP \\
\hline Size (nm) & 1000 & 30 \\
Settling & $<5 \%$ & None \\
Peptides per particle & $3.0 \times 10^{5}$ & $20-30$ \\
R2 ( $\left.\mathrm{sec}^{-1} / \mathrm{mM} \mathrm{Fe}\right)$ & 43 & 50 \\
$\mathrm{M}(\mathrm{emu} / \mathrm{g} \mathrm{Fe})$ & 105 & 86.6 \\
Fe atoms per particle & $2.8 \times 10^{9}$ & $8000^{[a]}$ \\
Particle Conc. @ & $5.1 \times 10^{-15}$ & $2.8 \times 10^{-9}$ \\
T2=100 msec, (M) & & \\
\hline$[a]$ From[22]. & &
\end{tabular}




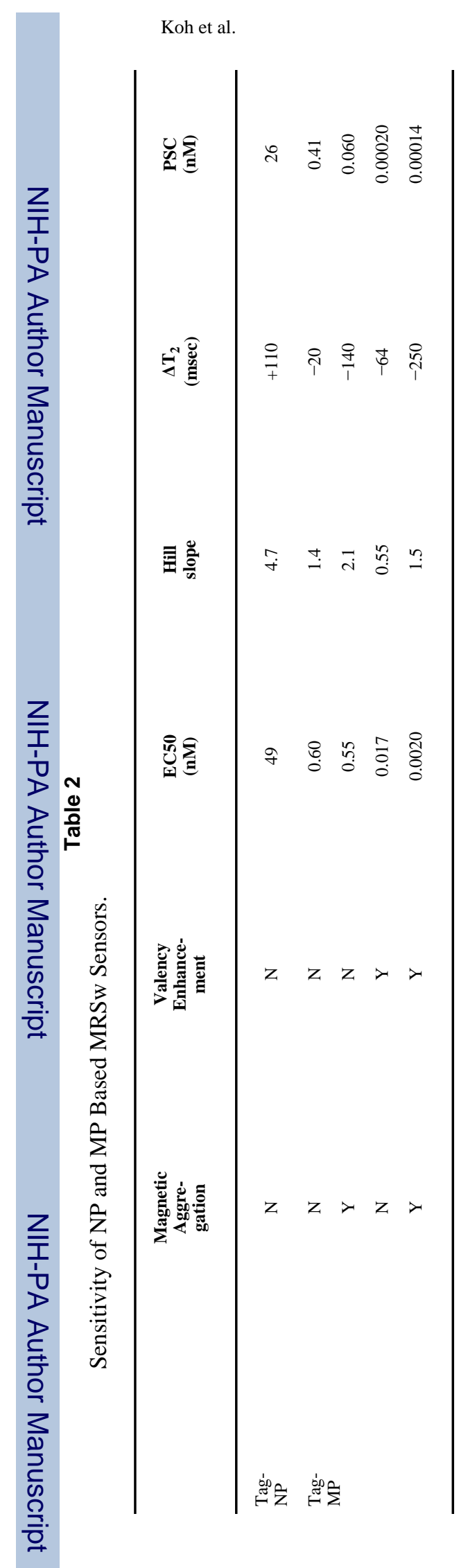

Page 8 